\title{
The Rupture Degree of Graphs with $k$-Tree
}

\author{
Yinkui Li, Qingning Wang, Xiaoling Wang \\ College of Mathematics and Statistics, Qinghai Nationalities University, Xining, China \\ Email: lyk463@163.com
}

Received 14 February 2016; accepted 19 April 2016; published 22 April 2016

Copyright $(2016$ by authors and Scientific Research Publishing Inc.

This work is licensed under the Creative Commons Attribution International License (CC BY).

http://creativecommons.org/licenses/by/4.0/

(c) $\underset{\mathrm{EY}}{\mathrm{EY}}$ Open Access

\section{Abstract}

A $k$-tree of a connected graph $G$ is a spanning tree with maximum degree at most $k$. The rupture degree for a connected graph $G$ is defined by

$r(G)=\max \{\omega(G-X)-|X|-m(G-X): X \subset V(G), \omega(G-X)>1\}$, where $m(G-X)$ and $\omega(G-X)$, respectively, denote the order of the largest component and number of components in $G-X$. In this paper, we show that for a connected graph $G$, if $r(G) \leq(k-3)|X|-m(G-X)+2$ for any cut-set $X \subset V(G)$, then $G$ has a $k$-tree.

\section{Keywords}

The Rupture Degree, $k$-Tree, Induced Graph

\section{Introduction}

Throughout this paper, We consider only finite undirected graphs without loops and multiple edges. A graph $G=(V, E)$ always means a simple connected graph with vertex set $V(G)$ and edge set $E(G)$. Let $\Delta$ denote the maximum degree of $G$, and $G[S]$ denote the subgraph of $G$ induced by a subset $S$ of $V(G)$. We by $d_{G}(v)$ denote the degree of a vertex $v$ in a graph $G$ and $N_{G}(v)$ the neighbor vertex set of $v$. Further for a nonempty subset $S$ of $V(G)$, we put $d_{G}(S)=\sum_{u \in S} d_{G}(u)$ and $N_{i}(S)=\left\{v \in V(G) \| N_{G}(v) \cap S \mid=i\right\}$.

A $k$-tree of a connected graph $G$ is a spanning tree with maximum degree $k$. Clearly, if $k=2$, it reduces to that of a Hamiltonian path in $G$. Since every tree with maximum degree $\Delta$ has a $\Delta$-tree, thus here we doesn't consider trees.

A nonempty set $S$ of independent vertices of $G$ is called a frame of $G$, if $G-S^{\prime}$ is connected for any $S^{\prime} \subseteq S$. If $|S|=k$ then $S$ is called a $k$-frame.

In [1] and [2], Win, Aung and Kyaw gave the ore-type condition and Fan-type condition for $k$ tree as fellows (Theorem A and B). 
Theorem A. If $d_{G}(S) \geq n-1$ for every independent set $S$ of $k$ vertices of graph $G$, then $G$ has a $k$ tree.

Theorem B. Let $V^{\star}=\left\{v \in V(G) \mid d_{G}(v)<\frac{n-1}{k}\right\}$ and suppose, either $V^{\star}=\varnothing$ or $G\left[V^{\star}\right]$ is a complete graph, then $G$ has a $k$ tree.

Further, Kyaw in [3] gave a stronger result for $k$ tree as theorem C.

Theorem C. Let $G$ be a connected graph and $k(\geq 2)$ an integer. If $d_{G}(S)+\sum_{i=2}^{k+1}(k-i) N_{i}(S) \geq n-1$ for every $k+1$-frame $S$ in $G$, then $G$ has a $k$ tree.

The rupture degree of a graph $G$ is introduced in [4], which is an important parameter for measuring the structure characteristics of the connected graph $G$ and defined as

$$
r(G)=\max \{\omega(G-X)-|X|-m(G-X): X \subset V(G), \omega(G-X)>1\}
$$

where $m(G-X)$ and $\omega(G-X)$, respectively, denote the order of the largest component and the number of components in $G-X$.

In this paper, we consider the rupture degree and existence of $k$-tree in a connected graph $G$ and thus give a new sufficient condition for a graph to have $k$ tree.

Any undefined terms can be found in the standard references on graph theory, including Bondy and Murty [5].

\section{Main Result}

Let $G$ be a connected graph and $k$ an integrity with $2 \leq k \leq \Delta$. Now, we by proving the following theorem to discuss the relationship between the rupture degree and existence of $k$-tree in graph $G$.

Theorem 1. Let $G$ be a connected graph but not a tree. If $r(G) \leq(k-3)|X|-m(G-X)+2$ for any cut-set $X \subset V(G)$, then $G$ has a k-tree.

Let $H$ be an induced subgraph of $G$ and with maximal order among all subgraphs containing $k$-tree, and let $A$ be a set adjacent to some vertices in $G$ but not in $V(H)$. Clearly, if $A=\varnothing$, then $G$ has $k$-tree. Now, we suppose that $r(G) \leq(k-3)|X|-m(G-X)+2$ for any cut-set $X \subset V(G)$ and $A$ is nonempty for connected graph $G$. We by finding a contradiction to prove the above theorem. Firstly, we prove the following claims.

Claim 1. Let $T$ be a $k$-tree of $H$. Then $d_{T}(x)=k$ for $x \in A$.

Proof. Let $T$ be a $k$-tree of $H$, which has maximal order among all the induced subgraphs of $G$ having a $k$-tree. On the contrary, suppose that if there exist some vertex $x \in A$ such that $d_{T}(x)<k$, then $H$ could be expanded by joining $x y$ for a neighbor $y$ of $x$ which is not in $H$. This is contradictive to the maximality of $H$. Thus $d_{T}(x)=k$ for any $x \in A$.

Let $T$ be a $k$-tree of $H$ and $x \in A$. Since $d_{T}(x)=k$, we suppose that $C_{1}, C_{2}, \cdots, C_{k}$ are all components of $T-x$.

Claim 2. If there exist an edge $u_{m} u_{n} \in E(H)$ for $u_{m} \in C_{m}, u_{n} \in C_{n}(1 \leq m \neq n \leq k)$, then $d_{T}\left(u_{m}\right)=k$ and $d_{T}\left(u_{n}\right)=k$.

Proof. Suppose that $d_{T}\left(u_{m}\right)<k$ (or $d_{T}\left(u_{n}\right)<k$ ) for $u_{m} \in C_{m}$ (or $u_{n} \in C_{n}$ ). Since $u_{m} u_{n} \in E(H)$, we may obtain a new $k$-tree $T^{*}$ from $T$ of $H$ by deleting one of the edges joining $x$ to components $C_{n}$ (or $C_{m}$ ) from $T$ and adding the edge $u_{m} u_{n}$ in $T$. Clearly, we obtain another $k$-tree of $H$ and in the latter $k$-tree, $x$ has degree less than $k$. Then $H$ could be expanded and this is contradictive to the maximality of $H$. So the conclusion holds.

Claim 3. Let $T$ be a k-tree of $H$ and $M$ is subset of $V(T)$ with degree $k$. Then $M$ is non-empty and satisfies the following property: Let $\mathbb{C}_{i}, 1 \leq i \leq r$, be the components of $T-M$. If for some $i$ and $j$ with $i \neq j$, the vertex $x_{i}$ of $\mathbb{C}_{i}$ is adjacent in $H$ to the vertex $x_{j}$ of $\mathbb{C}_{j}$, then $x_{i}$ and $x_{j}$ has degree $k$ and $x_{i}, x_{j} \in N \subset V(T)$.

Proof. Since $A$ is nonempty and for every $x \in A$ with $d_{T}(x)=k, M$ is non-empty. By Claim 2, the property holds with $r=k$ because the way in which we have picked the vertices $u_{n}$ or $u_{m}$ which go into $N$. Clearly, $M \cup N$ be a subset of the set of vertices of $T$ of degree $k$ in $T$. At the same time, we find that a vertex $v$ of $T$ adjacent to a vertex in $G$ but not in $V(H)$ is in $M \cup N$.

Let $T$ be a $k$-tree of $H$ with as many $k$ degree vertices as possible and $M, N$ be subsets of $V(T)$ with 
degree $k$ as above. Suppose that $\mathbb{C}_{i}$ is one component of $T-M$. If $N \cap V\left(\mathbb{C}_{i}\right)$ is nonempty, we select $y \in N \cap V\left(\mathbb{C}_{i}\right)$ and suppose that $\mathbb{C}_{i j}, 1 \leq i \leq s$, be the components of $T-M-\{y\}$.

Claim 4. For $m$ and $n$, with $1 \leq m \neq n \leq s$, if there exists a vertex $y_{m}$ of $\mathbb{C}_{i m}$ adjacent to a vertex $y_{n}$ of $\mathbb{C}_{i n}$ in H. Then $d_{T}\left(y_{m}\right)=k$ and $d_{T}\left(y_{n}\right)=k$.

Proof. Suppose that $d_{T}\left(y_{m}\right)<k$ (or $d_{T}\left(y_{n}\right)<k$ ) for $y_{m} \in \mathbb{C}_{i m}$ (or $y_{n} \in \mathbb{C}_{i n}$ ). Since $y_{m} y_{n} \in E(H)$, we may obtain a new $k$-tree $T^{* *}$ from $T$ of $H$ by deleting one of the edges joining $y \in N \cap V\left(\mathbb{C}_{i}\right)$ to components $\mathbb{C}_{i n}$ (or $\mathbb{C}_{i m}$ ) from $T$ and adding the edge $y_{m} y_{n} \in E(H)$ in $T$. Clearly, $y$ has degree less than $k$ in $k$-tree $T^{* *}$ of $H$. Then combine $y \in N \cap V\left(\mathbb{C}_{i}\right)$ we know this is contradictive to Claim 3. The conclusion holds.

By taking $T, M, N$ as the claims and let $|M \cup N|$ be maximal, then we obtain the follows claim as a straightforward consequence.

Claim 5. Let $T$ be a $k$-tree of $H$ with maximal number of $k$ degree vertices. Then, there is no edge of $H$ joining any components of $T-M \cup N$.

Proof. Given our choice of $T, M$ and $N$ as above, we derive a contradiction by assuming that there is an edge $y z$ of $H$ with endpoints $y$ and $z$ joining two components of $T-M \cup N$. If the path in $T$ joining $y$ and $z$ contains a vertex of $M$, then by claim 3, either $y$ or $z$ is in $N$ which is absurd. Then this path contains no vertex of $M$ and $y$ and $z$ are therefore in the same component $\mathbb{C}_{i}$ of $T-M$ for some $i$ with $1 \leq i \leq r$. Now let $w$ be a vertex of $N$ on the path in $T$ joining $y$ and $z$ and let $\mathbb{C}_{i j}, 1 \leq j \leq s$, be the components of $\mathbb{C}_{i}-\{w\}$. Now let $N_{0}$ be the set of all vertices of $\mathbb{C}_{i}-\{w\}$ having property of claim 4. Further, let $M^{*}=M \cup\{w\}$ and $N^{*}=N \cup N_{0}-\{w\}$. Since $N_{0}$ contains $y$ or $z$, claim 2 holds with $M^{*}$ and $N^{*}$ replacing $M$ and $N$, respectively. Moreover, $\left|M^{*} \cup N^{*}\right|$ is greater than $|M \cup N|$. But this contradicts to our choice of $T, M$ and $N$. This shows that there is no edge in $H$ joining any components of $T-M \cup N$.

Now we are ready for the proof of theorem 2.1.

The proof of theorem 2.1. Since $A$ is nonempty and thus $H$ is an induced proper subgraph of $G$, we have $\omega(G-M \cup N) \geq \omega(H-M \cup N)+1$. By claim 5 we know that $\omega(H-M \cup N)=\omega(T-M \cup N)$. At the same time, we know that $\omega(T-M \cup N)$ reaches minimum when $T[M \cup N]$ is itself a tree. Thus we have

$$
\begin{aligned}
\omega(G-M \cup N) & \geq \omega(H-M \cup N)+1=\omega(T-M \cup N)+1 \\
& \geq k|M \cup N|-2(|M \cup N|-1)+1=(k-2)|M \cup N|+3
\end{aligned}
$$

On the other hand, since $r(G) \leq(k-3)|X|-m(G-X)+2$ for any cut-set $X \subset V(G)$ we have

$$
\omega(G-X)-|X|-m(G-X) \leq(k-3)|X|-m(G-X)+2
$$

and,

$$
\omega(G-X) \leq(k-2)|X|+2
$$

This is a contradiction. Therefore $A$ is empty and the proof is completed.

\section{Acknowledgements}

We thank the editor and the referee for their comments. Research of Qingning Wang and Xiaoling Wang is funded by the National Science Foundation grant 11561056. This support is greatly appreciated.

\section{References}

[1] Win, S. (1975) Existenz von gerusten mit vorgeschriebenen maximalgrad in graphen. Adhandlungen aus den Mathematischen Seminor der Universitat Hamburg, 43, 263-267. http://dx.doi.org/10.1007/BF02995957

[2] Aung, M. and Kyaw, A. (1998) Maximal Trees with Bounded Maximum Degree in a Graph. Graphs and Combinatorics, 14, 209-221. http://dx.doi.org/10.1007/s003730050027

[3] Kyaw, A. (2001) A Sufficient Condition for a Graph to Have a k-Tree. Graphs and Combinatorics, 14, 113-121. http://dx.doi.org/10.1007/s003730170059

[4] Li, Y., Zhang, S. and Li, X. (2005) The Rupture Degree of Graphs. International Journal of Computer Mathematics, 82, 793-803. http://dx.doi.org/10.1080/00207160412331336062

[5] Bondy, J.A. and Murty, U.S.R. (1976) Graph Theory with Applications. Macmillan London and Elsevier, New York. http://dx.doi.org/10.1007/978-1-349-03521-2 